\title{
Substituição parcial do milho por sorgo granífero na alimentação de matrizes suínas primíparas nos períodos de puberdade e gestação
}

\author{
[Partial substitution of corn by sorghum in the feeding of primiparous female pigs during \\ puberty and pregnancy] \\ F.R.C. Moreira ${ }^{1}$, A.N. Costa ${ }^{2}$, T.D.D. Martins ${ }^{3}$, J.H.V. Silva ${ }^{3}$, \\ G.R.B. $\mathrm{Cruz}^{3}$, L.A.F. Pascoal \\ ${ }^{1}$ Aluno de pós-graduação/Universidade Federal da Paraíba - Areia, PB - IFRN - Apodi-RN \\ ${ }^{2}$ Bolsista DCR/CNPq - Fapern/UFRN \\ ${ }^{3} \mathrm{CCHSA} / \mathrm{UFPB}$ - Bananeiras, PB
}

\begin{abstract}
RESUMO
Avaliou-se o efeito da substituição parcial do milho por sorgo granífero na dieta de matrizes suínas híbridas durante a puberdade e a gestação. Foram utilizadas 25 leitoas, distribuídas em três tratamentos com porcentagens crescentes de sorgo granífero - 0\%, $25 \%$ e 50\% - em substituição ao milho. As fêmeas foram avaliadas durante as fases de pré-puberdade, puberdade e gestação, tendo sido mensurados o peso vivo (PV) e a idade à puberdade nas jovens e o PV e a espessura de toucinho (ET) nas fêmeas gestantes à cobrição e aos 30, 60, 90 e 110 dias de prenhez. Na fase de pré-puberdade, o ganho de peso diário diminuiu com o aumento da utilização do sorgo, e durante a puberdade não houve efeito dos tratamentos. Houve efeito linear da substituição sobre o PV aos 90 dias de gestação. Nos demais períodos, não foram encontrados efeitos significativos. Conclui-se que o sorgo granífero pode substituir o milho em até $50 \%$ na dieta de fêmeas suínas durante a puberdade e a gestação, sem comprometer as variáveis produtivas e reprodutivas.
\end{abstract}

Palavras-chave: alimento alternativo, fêmeas suínas, reprodução, suíno

\begin{abstract}
The effect of partial substitution of corn by sorghum on the diets of hybrid female pigs during puberty and pregnancy was evaluated. Twenty-five females were distributed in three treatments with increasing percentage of grain sorghum - 0\%, 25\% and 50\% - as a partial substitute for corn. The females were evaluated during the growth, puberty and pregnancy phases, measuring live weight (LW) and age at puberty in young females and LW and backfat thickness (BT) during gestation - at mating, 30, 60, 90 and 110 days of pregnancy. During the growth period, daily gain decreased with increased use of sorghum and there was no effect of treatments on puberty. For pregnancy, there was a linear effect on $L W$ at day 90. Thus, it was concluded that grain sorghum can replace corn up to $50 \%$ on the diet of female pigs during puberty, pregnancy and lactation without compromising the productivity and reproductive variables.
\end{abstract}

Keywords: alternative feed, female pigs, reproduction, swine

\section{INTRODUÇÃO}

O cenário atual da suinocultura estabelece como meta a redução de custos sem o declínio da produtividade dos animais. Nesse sentido, as

Recebido em 8 de novembro de 2011

Aceito em 7 de fevereiro de 2013

E-mail: faviano_moreira@hotmail.com

Apoio financeiro: CNPq/FAPERN/UFRN/BNB pesquisas com nutrição buscam ingredientes alternativos que associem preço acessível, oferta contínua e garantam bons índices de eficiência animal. A produtividade de uma granja de suínos pode ser avaliada pela sua eficiência reprodutiva, e o desempenho das matrizes é dependente de 
fatores, como genética, manejo, ambiente e nutrição, que influenciam diretamente a produtividade da empresa suinícola (Paiva et al., 2005).

As matrizes suínas, principalmente as primíparas, dependem de um aporte adequado de nutrientes para uma melhor produtividade ao longo de sua vida útil (Oelke et al., 2008). Dessa forma, para o uso de qualquer alimento alternativo, como o sorgo, é necessário aprofundar os conhecimentos sobre os efeitos biológicos desses alimentos nos animais.

O sorgo é tido como o quinto cereal mais importante no mundo e possui como vantagens a resistência à seca e o uso em solos arenosos $(\mathrm{Gu}$ et al., 2008). Apesar das vantagens agronômicas, o sorgo tem sido pouco utilizado nas dietas de não ruminantes, devido, em parte, ao conteúdo de tanino e ao menor teor de lisina de algumas variedades (Nyannor et al., 2007). Por essa razão, para os não ruminantes, recomenda-se o uso do sorgo com baixo teor de tanino (Gu et al., 2008). Segundo Whitaker e Carvalho (1997), a utilização do sorgo granífero como fonte alternativa de energia na alimentação animal tem mostrado resultados difíceis de serem comparados, em virtude da variabilidade na composição química dos cultivares e da diversidade de métodos para análise do teor de tanino. Quanto aos aspectos nutricionais, o sorgo apresenta nível de proteína bruta ligeiramente superior ao do milho, mas pode ser considerado equivalente em minerais e vitaminas e deficiente em gordura e em substâncias pigmentantes. Algumas variedades podem apresentar menores valores para aminoácidos básicos, como lisina, histidina e arginina, em comparação ao milho (Healy et al., 1994).

Com base nessas informações, objetivou-se estudar a substituição parcial do milho por sorgo granífero com baixo teor de tanino sobre o desempenho de matrizes suínas híbridas durante as fases de pré-puberdade, puberdade e gestação.

\section{MATERIAL E MÉTODOS}

Foram utilizadas 25 leitoas híbridas de linhagem comercial (Camborough F2 ${ }^{\circledR}$ ), a partir dos 56,56 $\pm 4,73 \mathrm{~kg}$, sendo avaliadas durante as fases de pré-puberdade, puberdade e gestação do primeiro ciclo reprodutivo. Os animais foram distribuídos em delineamento experimental inteiramente ao acaso, com três tratamentos, correspondentes a $0 \%(n=8), 25 \%(n=10)$ e $50 \%(n=7)$ de sorgo granífero em substituição ao milho.

As rações experimentais foram compostas principalmente por milho, sorgo, farelo de soja e suplementadas com vitaminas e minerais para atender as exigências nutricionais descritas por Rostagno et al. (2005) para cada fase experimental. O sorgo utilizado foi o genótipo IPA 7301011, considerado de baixo tanino (Tabosa et al., 1999). Nas fases pré-púbere e púbere (Tab. 1), as leitoas consumiram, em média, $2,5 \mathrm{~kg}$ de ração/dia. No período de gestação (Tab. 2), cada fêmea consumiu, em média, $2,0 \mathrm{~kg}$ de ração (primeiro ao $30^{\circ}$ dia), $2,2 \mathrm{~kg}\left(31^{\circ}\right.$ ao $85^{\circ}$ dia $), 2,5 \mathrm{~kg}\left(86^{\circ}\right.$ ao $110^{\circ}$ dia $) \mathrm{e}$ $2,0 \mathrm{~kg}\left(110^{\circ}\right.$ dia ao parto), distribuídos em dois arraçoamentos diários - manhã e tarde. Durante todo o período experimental, o fornecimento de água foi à vontade.

As fêmeas foram alojadas aos pares nas baias experimentais. As pesagens foram realizadas mensalmente, durante a fase pré-púbere e até as fêmeas atingirem a puberdade - primeiro estro. As fêmeas foram ainda pesadas à cobrição terceiro estro - e durante a gestação - aos $30^{\circ}$, $60^{\circ}, 90^{\circ}, 110^{\circ}$ dia de prenhez.

A espessura do toucinho (ET) foi mensurada aos 60, 90 e 110 dias de gestação por meio de aparelho de ultrassom (Modelo MTU-100, Microem Produtos Médicos Ltda., SP), entre a penúltima e a última costela a $6,5 \mathrm{~cm}$ da linha média dorsal, nos lados direito e esquerdo, para o cálculo da média dos valores.

Com base nas pesagens e mensurações de ET, foi avaliada a variação de peso durante a fase prépúbere e a variação de peso e ET durante a gestação - da cobertura ao $60^{\circ}$ dia de gestação e da cobertura ao $110^{\circ}$ dia de gestação.

As fêmeas foram estimuladas para o aparecimento de estro a partir dos 150 dias de idade, com auxílio de um varrão adulto, por meio de contato tátil duas vezes ao dia, por cerca de 10 minutos. As leitoas atingiram a idade de puberdade (dias) quando apresentaram sinais de estro e foram fertilizadas no terceiro estro. 
Tabela 1. Composição alimentar ( $\mathrm{g} / \mathrm{kg}$ de matéria natural) e valores calculados $(\mathrm{g} / \mathrm{kg})$ das rações experimentais para a fase pré-pubere $1(50-70 \mathrm{~kg})$ e pré-púbere 2 (70-100kg de peso vivo) *

\begin{tabular}{|c|c|c|c|c|c|c|}
\hline \multirow{3}{*}{ Ingrediente } & \multicolumn{6}{|c|}{ Níveis de substituição do milho por sorgo granífero (\%) } \\
\hline & \multicolumn{3}{|c|}{ Pré-púbere 1} & \multicolumn{3}{|c|}{ Pré-púbere 2} \\
\hline & 0 & 25 & 50 & 0 & 25 & 50 \\
\hline Milho & 604,57 & 453,43 & 302,28 & 736,96 & 552,72 & 368,48 \\
\hline Sorgo de baixo tanino & 0,00 & 151,14 & 302,28 & 0,00 & 184,24 & 368,48 \\
\hline Farelo de soja (45\%) & 270,35 & 260,72 & 251,10 & 210,12 & 198,90 & 187,68 \\
\hline Óleo de soja & 42,11 & 42,22 & 42,34 & 14,11 & 14,15 & 14,20 \\
\hline Glúten de milho (60\%) & 0,00 & 6,15 & 12,30 & 0,00 & 7,11 & 14,22 \\
\hline Fosfato bicálcico & 9,85 & 9,82 & 9,78 & 8,17 & 8,12 & 8,07 \\
\hline Calcário & 5,26 & 5,32 & 5,37 & 4,78 & 4,86 & 4,94 \\
\hline Sal comum & 3,36 & 3,41 & 3,46 & 3,01 & 3,07 & 3,14 \\
\hline L-lisina HCL & 3,50 & 3,82 & 4,14 & 2,39 & 2,78 & 3,18 \\
\hline L-treonina & 1,33 & 1,37 & 1,41 & 0,76 & 0,81 & 0,86 \\
\hline DL-metionina & 1,28 & 1,31 & 1,34 & 0,31 & 0,36 & 0,40 \\
\hline Premix vitamínico ${ }^{1}$ & 4,00 & 4,00 & 4,00 & 4,00 & 4,00 & 4,00 \\
\hline Premix mineral $^{2}$ & 0,50 & 0,50 & 0,50 & 0,50 & 0,50 & 0,50 \\
\hline Inerte $^{3}$ & 53,79 & 56,69 & 59,60 & 17,28 & 20,77 & 24,25 \\
\hline BHT (antifúngico) ${ }^{4}$ & 0,10 & 0,10 & 0,10 & 0,10 & 0,10 & 0,10 \\
\hline Total (g) & 1000,00 & 1000,00 & 1000,00 & 1000,00 & 1000,00 & 1000,00 \\
\hline \multicolumn{7}{|l|}{ Valor calculado } \\
\hline Energia metabolizável (Mcal/kg) & 3,230 & 3,230 & 3,230 & 3,230 & 3,230 & 3,230 \\
\hline Proteína bruta (g/kg) & 180,0 & 180,0 & 180,0 & 162,0 & 162,0 & 162,0 \\
\hline Cálcio $(\mathrm{g} / \mathrm{kg})$ & 5,51 & 5,51 & 5,51 & 4,84 & 4,84 & 4,84 \\
\hline Fósforo disponível (g/kg) & 2,82 & 2,82 & 2,80 & 2,48 & 2,48 & 2,48 \\
\hline Lisina total $(\mathrm{g} / \mathrm{kg})$ & 11,77 & 11,77 & 11,77 & 9,75 & 9,75 & 9,75 \\
\hline Metionina+cistina total $(\mathrm{g} / \mathrm{kg})$ & 6,94 & 6,94 & 6,94 & 5,85 & 5,85 & 5,85 \\
\hline Metionina total $(\mathrm{g} / \mathrm{kg})$ & 4,06 & 4,10 & 4,13 & 2,93 & 2,98 & 3,04 \\
\hline Treonina total $(\mathrm{g} / \mathrm{kg})$ & 8,12 & 8,12 & 8,12 & 6,92 & 6,92 & 6,92 \\
\hline Triptofano total $(\mathrm{g} / \mathrm{kg})$ & 2,12 & 2,12 & 2,12 & 1,85 & 1,85 & 1,85 \\
\hline Sódio $(\mathrm{g} / \mathrm{kg})$ & 1,70 & 1,70 & 1,70 & 1,60 & 1,60 & 1,60 \\
\hline Gordura $(\mathrm{g} / \mathrm{kg})$ & 66,33 & 65,58 & 64,83 & 41,13 & 40,17 & 39,22 \\
\hline Fibra bruta $(\mathrm{g} / \mathrm{kg})$ & 27,79 & 27,71 & 27,63 & 28,03 & 27,89 & 27,75 \\
\hline
\end{tabular}

${ }^{\mathrm{T}}$ Quantidade por kg do produto: vit. A 225.000 UI, vit. $\mathrm{D}_{3} 37.500 \mathrm{UI}$, vit. E $1.500 \mathrm{mg}$, vit. K $75 \mathrm{mg}$, vit. B12 625mg, niacina $1.000 \mathrm{mg}$, ácido pantotênico $500 \mathrm{mg}$, ácido fólico $65 \mathrm{mg}$, biotina $6,75 \mathrm{mg}$, colina $8.400 \mathrm{mg}$, piridoxina $100 \mathrm{mg}$, riboflavina $150 \mathrm{mg}$, tiamina $32,5 \mathrm{mg} .{ }^{2}$ Quantidade por $\mathrm{kg}$ do produto: cálcio $215 \mathrm{~g}$, cobre $450 \mathrm{mg}$, ferro $2.750 \mathrm{mg}$, fósforo $85 \mathrm{mg}$, flúor $850 \mathrm{mg}$, iodo $17,5 \mathrm{mg}$, manganês $1.250 \mathrm{mg}$, selênio $7,5 \mathrm{mg}$, sódio $49 \mathrm{mg}$, zinco $2.750 \mathrm{mg}$, cromo $5 \mathrm{mg}$, bacitracina de zinco $1.000 \mathrm{mg} .{ }^{3}$ Inerte $=$ areia lavada; ${ }^{4} \mathrm{BHT}=$ Butil-hidroxitolueno. $*$ Valores propostos por Rostagno et al. (2005). 
Tabela 2. Composição alimentar ( $\mathrm{g} / \mathrm{kg}$ de matéria natural) e valores calculados $(\mathrm{g} / \mathrm{kg})$ das rações experimentais para a fase de gestação para matrizes suínas*

\begin{tabular}{|c|c|c|c|}
\hline \multirow{3}{*}{ Ingrediente } & \multicolumn{3}{|c|}{ Níveis de substituição do milho por sorgo granífero (\%) } \\
\hline & \multicolumn{3}{|c|}{ Gestação } \\
\hline & 0 & 25 & 50 \\
\hline Milho & 452,61 & 339,46 & 226,30 \\
\hline Sorgo baixo tanino & 0,00 & 113,15 & 226,30 \\
\hline Farelo de soja (45\%) & 167,74 & 160,58 & 153,40 \\
\hline Farelo de trigo & 150,00 & 150,00 & 150,00 \\
\hline Amido & 142,67 & 146,49 & 150,33 \\
\hline Óleo de soja & 17,03 & 15,49 & 13,94 \\
\hline Glúten de milho (60\%) & 0,00 & 4,53 & 9,08 \\
\hline Fosfato bicálcico & 14,51 & 14,48 & 14,46 \\
\hline Calcário & 6,86 & 6,90 & 6,94 \\
\hline Sal comum & 3,78 & 3,82 & 3,85 \\
\hline L-lisina HCL & 1,10 & 1,34 & 1,58 \\
\hline L-treonina & 1,60 & 1,63 & 1,67 \\
\hline DL-metionina & 0,00 & 0,02 & 0,05 \\
\hline Premix vitamínico ${ }^{1}$ & 4,00 & 4,00 & 4,00 \\
\hline Premix mineral $^{2}$ & 0,50 & 0,50 & 0,50 \\
\hline Inerte $^{3}$ & 37,50 & 37,50 & 37,50 \\
\hline BHT (antifúngico) ${ }^{4}$ & 0,10 & 0,10 & 0,10 \\
\hline Total (g) & 1000,00 & 1000,00 & 1000,00 \\
\hline \multicolumn{4}{|l|}{ Valor calculado } \\
\hline Energia metabolizável (Mcal/kg) & 3,020 & 3,020 & 3,020 \\
\hline Proteína bruta $(\mathrm{g} / \mathrm{kg})$ & 14,30 & 14,30 & 14,30 \\
\hline Cálcio (g/kg) & 0,710 & 0,710 & 0,710 \\
\hline Fósforo disponível (g/kg) & 0,380 & 0,380 & 0,380 \\
\hline Lisina total $(\mathrm{g} / \mathrm{kg})$ & 0,760 & 0,760 & 0,700 \\
\hline Metionina+cistina total $(\mathrm{g} / \mathrm{kg})$ & 0,460 & 0,460 & 0,600 \\
\hline Metionina total $(\mathrm{g} / \mathrm{kg})$ & 0,219 & 0,222 & 0,225 \\
\hline Treonina total $(\mathrm{g} / \mathrm{kg})$ & 0,680 & 0,680 & 0,680 \\
\hline Triptofano total $(\mathrm{g} / \mathrm{kg})$ & 0,166 & 0,166 & 0,166 \\
\hline Gordura (g/kg) & 4,015 & 3,798 & 3,580 \\
\hline Fibra bruta $(\mathrm{g} / \mathrm{kg})$ & 3,221 & 3,215 & 3,209 \\
\hline
\end{tabular}

${ }^{1}$ Quantidade por kg do produto: vit. A $225.000 \mathrm{UI}$, vit. $\mathrm{D}_{3} 37.500 \mathrm{UI}$, vit. E $1.500 \mathrm{mg}$, vit. K $75 \mathrm{mg}$, vit. B12 625mg, niacina $1.000 \mathrm{mg}$, ácido pantotênico $500 \mathrm{mg}$, ácido fólico $65 \mathrm{mg}$, biotina $6,75 \mathrm{mg}$, colina $8.400 \mathrm{mg}$, piridoxina $100 \mathrm{mg}$, riboflavina $150 \mathrm{mg}$, tiamina $32,5 \mathrm{mg}$. ${ }^{2}$ Quantidade por $\mathrm{kg}$ do produto: cálcio $215 \mathrm{~g}$, cobre $450 \mathrm{mg}$, ferro $2.750 \mathrm{mg}$, fósforo $85 \mathrm{mg}$, flúor $850 \mathrm{mg}$, iodo $17,5 \mathrm{mg}$, manganês $1.250 \mathrm{mg}$, selênio $7,5 \mathrm{mg}$, sódio $49 \mathrm{mg}$, zinco $2.750 \mathrm{mg}$, cromo $5 \mathrm{mg}$, bacitracina de zinco $1.000 \mathrm{mg} .{ }^{3}$ Inerte $=$ areia lavada; ${ }^{4} \mathrm{BHT}=$ Butil-hidroxitolueno. $*$ Valores propostos por Rostagno et al. (2005). 
Os parâmetros estudados no experimento foram submetidos à análise de variância e de regressão por meio dos polinômios ortogonais. Em todas as análises estatísticas, utilizou-se o procedimento GLM no programa estatístico SAS (Statistical..., 2005) e as recomendações de Reis (2003).

\section{RESULTADOS E DISCUSSÃO}

Os dados constantes da Tab. 3 evidenciam que o peso das leitoas aos 120 e 150 dias não diferiu $(\mathrm{P}>0,05)$ entre os tratamentos, todavia, para o ganho de peso diário (GPD) na fase pré-púbere, observou-se uma redução linear $(y=835,57-$ $\left.72,91 \mathrm{x} ; \mathrm{R}^{2}=0,95\right)$ dessa variável com o aumento da substituição do milho por sorgo. Para idade, peso e GPD das leitoas não houve diferença $(\mathrm{P}>0,05)$ entre tratamentos à puberdade. Para todas as variáveis não foi observado efeito quadrático.
Na fase pré-púbere, o GPD das fêmeas nos tratamentos não influenciou o aparecimento da puberdade, embora o GPD do tratamento controle tenha apresentado um maior valor $(\mathrm{P}<0,05)$ em relação ao tratamento com $50 \%$ de sorgo em substituição ao milho. Segundo Beltranena et al. (1991), o GPD só influenciará a idade à puberdade quando for abaixo dos limites mínimos. De acordo com esses autores e a Agroceres (2003), GPD entre 600 e $660 \mathrm{~g}$ é suficiente para uma puberdade precoce. Como todos os tratamentos apresentaram GPD dentro dos parâmetros recomendados, não ocorreram diferenças na idade à puberdade. Kummer et al. (2009) também não observaram diferenças na taxa de prenhez, no número de ovulações e no número de embriões para porcas com GPD de 577 e $724 \mathrm{~g} / \mathrm{dia}$ entre o nascimento e os 144 dias de idade.

Tabela 3. Peso (kg), ganho de peso diário (g) e idade (dias) nas fases pré-púbere e púbere de leitoas alimentadas com rações contendo 0,25 ou $50 \%$ de sorgo granífero na dieta em substituição ao milho

\begin{tabular}{lccccc}
\multirow{2}{*}{ Variável } & \multicolumn{4}{c}{ Substituição do milho por sorgo granífero $(\%)$} & Efeito \\
\cline { 2 - 6 } & 0 & 25 & 50 & CV $(\%)$ & Linear \\
\hline Peso aos 120 dias (kg) & 57,4 & 55,9 & 56,6 & 8,6 & $\mathrm{~ns}$ \\
Peso aos 150 dias (kg) & 80,6 & 76,1 & 75,4 & 9,5 & $\mathrm{~ns}$ \\
Ganho de peso diário $(\mathrm{g})$ & 773,0 & 673,0 & 627,0 & 16,5 & $* *$ \\
\hline & \multicolumn{5}{c}{ Puberdade } \\
\hline Idade à puberdade (dias) & 160,0 & 165,0 & 161,4 & 5,2 & $\mathrm{~ns}$ \\
Peso vivo à puberdade $(\mathrm{kg})$ & 92,5 & 90,0 & 89,4 & 9,3 & $\mathrm{~ns}$ \\
Ganho de peso diário $(\mathrm{g}) *$ & 870,6 & 780,6 & 802,3 & 17,6 & $\mathrm{~ns}$ \\
\hline
\end{tabular}

* Ganho de peso diário até atingir a puberdade (primeiro estro).

** $\mathrm{y}=835,57-72,91 \times\left(\mathrm{R}^{2}=0,95\right)$.

Amaral Filha et al. (2009) analisaram a influência da taxa de crescimento e o início de exposição ao macho em diferentes momentos e encontraram que porcas com maior ganho de peso (entre 726 e $830 \mathrm{~g} / \mathrm{dia}$ ) podem ser expostas em idades mais precoces (130-149 dias), consequentemente antecipando a monta sem comprometer o desempenho reprodutivo. Todavia, caso o peso e o ganho de peso sejam baixos e a estimulação ao estro inicie precocemente, orienta-se que a cobertura ocorra no quarto estro.

Rodrigues et al. (2002), ao analisarem o ganho de peso e a conversão alimentar de fêmeas entre 30 e $90 \mathrm{~kg}$ de peso vivo, encontraram resultados similares quando avaliaram dietas à base de sorgo ou milho.
Conforme mostra a Tab. 3, o aparecimento do primeiro estro variou entre 160 e 165 dias de idade, tendo a exposição das fêmeas ao varrão iniciada aos 150 dias. Pesquisas realizadas por Gaughan et al. (1997) com leitoas pré-púberes, alimentadas com rações contendo $100 \%$ de sorgo de baixo tanino e estimuladas pelo macho a partir dos 165 dias de idade, mostraram que as fêmeas atingiram a puberdade aos 174 dias de idade. Tal diferença pode ser explicada pela variação no início da exposição das fêmeas ao macho.

Quanto à gestação (Tab. 4), foi encontrado efeito linear apenas para o peso das fêmeas aos 90 dias de prenhez $\left(y=193,01-7,54 x ; R^{2}=0,92\right)$. Não foi observado efeito da substituição do milho pelo sorgo em relação ao peso e à espessura de toucinho nos demais estágios de gestação. Também não foi observado efeito quadrático. 
Substituição parcial do milho...

Tabela 4. Peso $(\mathrm{kg})$ e espessura de toucinho $(\mathrm{mm})$ na gestação de fêmeas suínas alimentadas com rações com 0,25 ou $50 \%$ de sorgo granífero em substituição ao milho

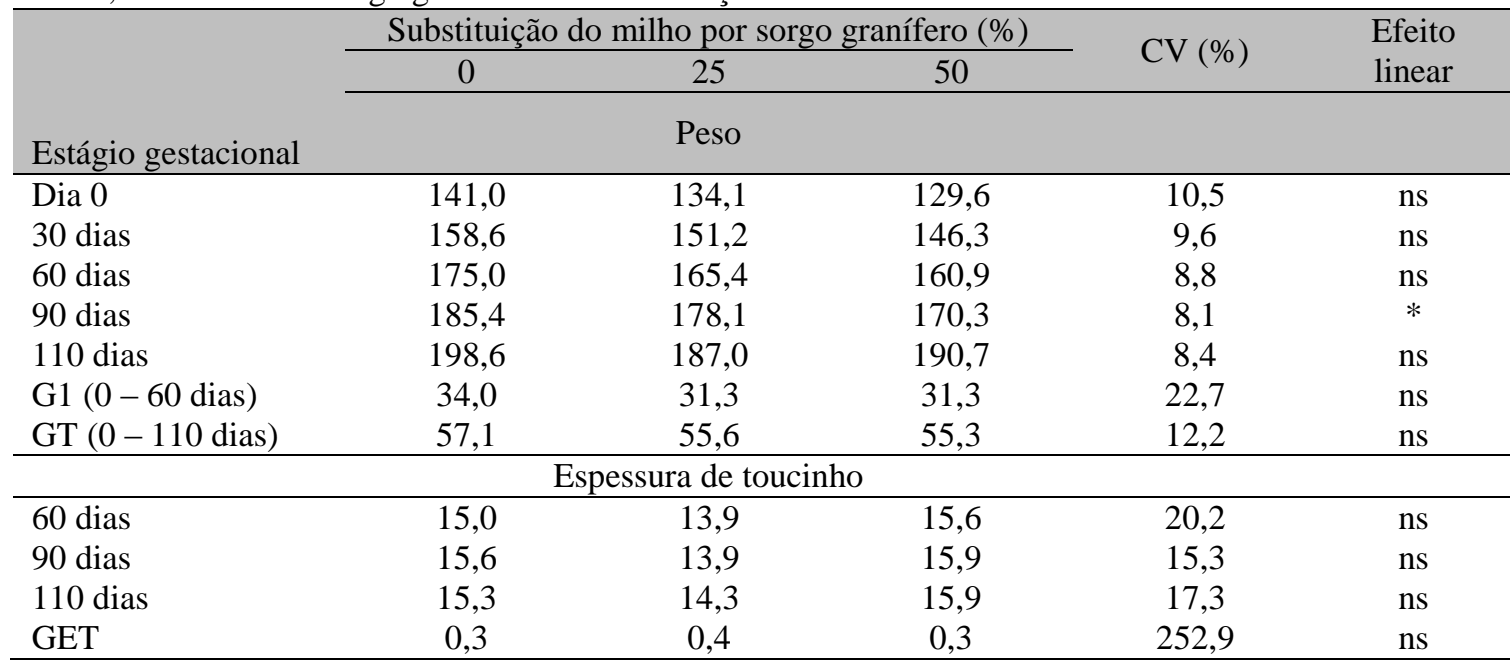

G1 - variação de peso entre 0 e 60 dias de gestação; GT - variação de peso entre 0 e 110 dias de gestação; GET variação da espessura de toucinho entre 60 e 110 dias de gestação.

$* \mathrm{y}=193,01-7,54 \mathrm{x}\left(\mathrm{R}^{2}=0,92\right)$.

Com relação ao ganho de peso durante a gestação (Tab. 4), em todos os tratamentos as fêmeas ganharam mais peso do que os 45,0 e $49,0 \mathrm{~kg}$ preconizados por Verstegen et al. (1987) e García-Castillo et al. (2004), respectivamente. Provavelmente as diferenças foram devido à genética e às dietas experimentais utilizadas nos diversos experimentos. Em todos os tratamentos, o maior ganho de peso ocorreu entre zero e 60 dias de gestação (G1), fato que não condiz com o maior crescimento dos leitões. Segundo Lima et al. (2006), o ganho maior de peso na primeira metade da gestação pode ser explicado pelo fato de as fêmeas serem primíparas e não terem atingido o peso adulto, depositando ainda tecido de reserva. Isso indica que as linhagens atuais podem ser mais propensas ao catabolismo lactacional severo (Mellagi et al., 2010).

Em porcas gestantes alimentadas com dietas à base de sorgo como fonte energética, Louis et al. (1991) não encontraram diferenças significativas para as fêmeas alimentadas com milho, o que indica que os dois cereais apresentaram desempenho semelhante.

\section{CONCLUSÕES}

A substituição de até $50 \%$ do milho por sorgo granífero na dieta de matrizes suínas primíparas, durante as fases de puberdade e gestação, pode representar uma alternativa viável à produção sustentável de suínos em clima tropical.

\section{REFERÊNCIAS}

AGROCERES PIC. Gilt Management Guide, 2003. Disponível em: http://www.agrocerespic.com.br Acessado em: 10 mar. 2009.

AMARAL FILHA, W.S.; BERNARDI, M.L.; WENTZ, I. et al. Growth rate and age at boar exposure as factors influencing gilt puberty. Livest. Sci., v.120, p.51-57, 2009.

BELTRANENA, E.; AHERNE, F.X.; FOXCROFT, G.R. et al. Effects of pre and post pubertal feeding on production traits at first and second estrus. J. Anim. Sci., v.69, p.886-893, 1991.

GARCÍA-CASTILLO， R.F.; GUTIÉRREZBAÑUELOS, H.; MELLADO-BOSQUE, M. et al. Cromo L-metionina en dietas basadas en sorgo y soya en cerdas primerizas. Rev. Agrar. Nueva Epoca, v.1, p.6-11, 2004. 
GAUGHAN, J.B.; CAMERON, R.D.; DRYDEN, G.M. et al. Effect of body composition at selection on reproductive development in Large White gilts. J. Anim. Sci., v.75, p.1764-1772, 1997.

GU, L.; HOUSE, S.E.; ROONEY, L.W. et al. Sorghum extrusion increases bioavailability of catechins in wealing pigs. J. Agric. and Food Chemistry, v.56, p.1283-1288, 2008.

HEALY, B.J.; HANCOCK, J.D.; KENNEDY, G.A. et al. Optimum particle size of corn and hard and soft sorghum for nursery pigs. J. Anim. Sci., v.72, p.2227-2236, 1994.

KUMMER, R.; BERNARDI, M.L.; SCHENKEL, A.C. et al. Reproductive performance of gilts with similar age but with different growth rate at the onset of puberty stimulation. Reprod. Dom. Anim., v.44, p.255-259, 2009.

LIMA, K.R.S.; FERREIRA, A.S.; MANNO, M.C. et al. Níveis de proteína bruta na dieta e desempenho reprodutivo de fêmeas primíparas em gestação. Rev. Bras. Zootec., v.35, p.785791, 2006.

LOUIS, G.F.; LEWIS, A.J.; PEO, E.R. Feeding value of grain sorghum for the lactating sow. $J$. Anim. Sci., v.69, p.223-229, 1991.

MELLAGI, A.P.G.; ARGENTI, L.E.; FACCIN, J.E.G. et al. Aspectos nutricionais de matrizes suínas durante a lactação e o impacto na fertilidade. Acta Scient. Veterin., v.38, p.181209, 2010.

NYANNOR, E.K.D.; ADEDOKUN, S.A.; HAMAKER, B.R. et al. Nutritional evaluation of high-digestible sorghum for pigs and broiler chicks. J. Anim. Sci., v.85, p.196-203, 2007.
OELKE, C.A.; DAHLKE, F.; BELTRANI, O.C. et al. Níveis de lisina digestível em dietas para fêmeas suínas primíparas em lactação. Acta Scient. Anim. Sci., v.30, p.299-306, 2008.

PAIVA, F.P.; DONZELE, J.L.; OLIVEIRA, R.F.M. et al. Lisina em rações para fêmeas suínas primíparas em lactação. Rev. Bras. Zootec., v.34, p.1971-1979, 2005.

REIS, J.C. Estatística aplicada à pesquisa em ciência veterinária. 1.ed. Olinda: JCR, 2003. $651 \mathrm{p}$.

RODRIGUES, P.B.; FREITAS, R.T.F.; FIALHO, E.T. et al. Digestibilidade dos nutrientes e desempenho de suínos em crescimento e terminação alimentados com rações à base de milho e sorgo suplementadas com enzimas. Rev. Bras. Milho Sorgo, v.1, p.91-100, 2002.

ROSTAGNO, H.S.; ALBINO, L.F.T.; DONZELE, J.L. et al. Tabelas brasileiras para aves e suínos - composição de alimentos e exigências nutricionais. 2.ed. Viçosa: UFV, v.1., 2005. $186 \mathrm{p}$.

STATISTICAL analysis system - SAS. User's guide. Version 9.0. Cary: 2005. (CD-ROM).

VERSTEGEN, M.W.A.; VERHAGEN, J.M.F.; DEN HARTOG, L.A. Energy requirements of pigs during pregnancy: a review. Livest. Produc. Sci., v.16, p.75-89, 1987.

WHITAKER, H.M.A.; CARVALHO, R.L. Substituição do milho pelo sorgo em rações para equinos. Rev. Bras. Zootec., v.26, p.139-143, 1997. 